Supporting Information

\title{
The racemic synthesis of an intermediate for the formal synthesis of madindoline A and B
}

\author{
Qing Li, Fenfen Xiao, Yunxia Wang, ${ }^{*}$ Xiangdong $\mathrm{Hu}^{*}$ \\ School of Chemistry \& Material Science, Key Laboratory of Synthetic and Natural Functional \\ Molecule Chemistry of Ministry of Education of China, Northwest University, Xi'an 710127, China \\ e-mail: wyx27210@nwu.edu.cn; xiangdonghu@nwu.edu.cn
}

Table of Contents

Experimental for Synthesis of Compounds............................S2-S4

${ }^{1} \mathrm{H}$ and ${ }^{13} \mathrm{C}$ NMR Spectra of Compounds...............................S5-S12 
3-n-Butyl-4-methylcyclobut-3-ene-1,2-dione (16). A solution of $\mathrm{CH}_{3} \mathrm{Li}\left(1.6 \mathrm{M}\right.$ in $\mathrm{Et}_{2} \mathrm{O}, 3.4 \mathrm{~mL}$, $5.5 \mathrm{mmol}$ ) was added dropwise to a solution of diisopropyl squarate $(\mathbf{1 7 b})(990 \mathrm{mg}, 5.0 \mathrm{mmol})$ in THF (20 mL) at $-78^{\circ} \mathrm{C}$ under argon. After $5 \mathrm{~min}$ of stirring, a solution of $n$-BuLi (2.4 M in hexane, $2.5 \mathrm{~mL}, 6.0 \mathrm{mmol}$ ) was added dropwise to the system. $10 \mathrm{~min}$ later, the reaction was quenched with saturated ammonium chloride solution, and extracted with EtOAc. The organic layer was washed with water, brine, and dried over $\mathrm{Na}_{2} \mathrm{SO}_{4}$. Removal of the solvent, then a solution of concentrated hydrochloric acid in $\mathrm{CH}_{2} \mathrm{Cl}_{2}$ was added to a solution of the intermediate in $\mathrm{CH}_{2} \mathrm{Cl}_{2}$ and controlled $\mathrm{pH}=3$, stirred at room temperature about $30 \mathrm{~min}$, and then the mixture was dried over $\mathrm{Na}_{2} \mathrm{SO}_{4}$. Removal of the solvent and flash chromatography of the residue on silica gel $(\mathrm{PE} / \mathrm{EtOAC}=10 / 1)$ afforded the $16(600 \mathrm{mg}, 79 \%)$ brown oil. $R_{f}=0.4(\mathrm{PE} / \mathrm{EtOAC}=4 / 1) .{ }^{1} \mathrm{H} \mathrm{NMR}$ $\left(400 \mathrm{MHz}, \mathrm{CDCl}_{3}\right): \delta 2.74(\mathrm{t}, J=8 \mathrm{~Hz}, 2 \mathrm{H}), 2.35(\mathrm{~s}, 3 \mathrm{H}), 1.67-1.75(\mathrm{~m}, 2 \mathrm{H}), 1.37-1.43(\mathrm{~m}, 2 \mathrm{H}), 0.96(\mathrm{t}$, $J=8 \mathrm{~Hz}, 3 \mathrm{H}) .{ }^{13} \mathrm{CNMR}\left(100 \mathrm{MHz}, \mathrm{CDCl}_{3}\right): \delta 203.7,199.7,199.4,199.2,27.9,26.2,22.8,13.6,11.2$. HRMS $\left(E S I^{+}\right) \mathrm{m} / z:[\mathrm{M}+\mathrm{Na}]^{+}$calcd for $\mathrm{C}_{9} \mathrm{H}_{12} \mathrm{NaO}_{2}, 175.0730$; found, 175.0733 .

4-butyl-2-methoxycarbonyl-2,5-dimethylcyclopent-4-ene-1,3-dione (14b). colorless oil. (146 $\mathrm{mg}, 80 \%$ (based on the recovery of $23 \%$ of 16$)) R_{f}=0.6(\mathrm{PE} / \mathrm{EtOAC}=4 / 1) .{ }^{1} \mathrm{H} \mathrm{NMR}(400 \mathrm{MHz}$, $\left.\mathrm{CDCl}_{3}\right): \delta 3.67(\mathrm{~s}, 3 \mathrm{H}), 2.52(\mathrm{t}, J=8 \mathrm{~Hz}, 3 \mathrm{H}), 2.09(\mathrm{~s}, 3 \mathrm{H}), 1.48-1.55(\mathrm{~m}, 2 \mathrm{H}), 1.44(\mathrm{~s}, 3 \mathrm{H}), 1.33-1.39$ (m, 2H), $0.94(\mathrm{t}, J=8 \mathrm{~Hz}, 3 \mathrm{H}) .{ }^{13} \mathrm{C} \mathrm{NMR}\left(100 \mathrm{MHz}, \mathrm{CDCl}_{3}\right): \delta 199.7,199.4,167.1,159.9,155.5,57.4$, 53.2, 29.7, 24.1, 22.7, 15.3, 13.9, 9.7. $\mathrm{HRMS}\left(\mathrm{ESI}^{+}\right) \mathrm{m} / \mathrm{z}:[\mathrm{M}+\mathrm{Na}]^{+}$calcd for $\mathrm{C}_{13} \mathrm{H}_{18} \mathrm{NaO}_{4}, 261.1097$; found, 261.1090 .

((4-butyl-2-methoxycarbonyl-2,5-dimethylcyclopent-4-ene-1,3-diyl)bis(oxy))bis(tert-butyldim ethylsilane) (13): 14b (50 mg, $0.21 \mathrm{mmol}$ ) and cerous chloride heptahydrate (16 mg, $0.04 \mathrm{mmol}$ ) was dissolved in methanol $(5 \mathrm{~mL})$, the mixture was then cooled to $0^{\circ} \mathrm{C}$, and to the solution was 
added $\mathrm{NaBH}_{4}$ (32 mg, $0.84 \mathrm{mmol}$ ) slowly. The reaction mixture was warmed up to room temperature. After $3 \mathrm{~h}$, the reaction mixture was quenched with saturated ammonium chloride solution. The aqueous phase was extracted with $\mathrm{CH}_{2} \mathrm{Cl}_{2}(3 \times 20 \mathrm{~mL})$. The combined organic layer was washed with saturated brine and dried over $\mathrm{Na}_{2} \mathrm{SO}_{4}$. The solvent was removed under reduced pressure. Then tert- butyldimethylsilyl trifluoromethanesulfonate (TBSOTf) (172 mg, 1.1 $\mathrm{mmol}$ ) was added to the solution of the crude residue and triethylamine $(213 \mathrm{mg}, 2.1 \mathrm{mmol})$ in $\mathrm{CH}_{2} \mathrm{Cl}_{2}(5 \mathrm{~mL})$ at room temperature. After $1 \mathrm{~h}$, the reaction was quenched with water. The aqueous phase was extracted with $\mathrm{CH}_{2} \mathrm{Cl}_{2}$ and the combined organic layers were dried over $\mathrm{Na}_{2} \mathrm{SO}_{4}$. The solvent was removed under reduced pressure. The crude residue was purified by silica gel chromatography (PE/EtOAc, 100/1) to give $13(52 \mathrm{mg}, 53 \%)$ as a colorless oil. $R_{f}=0.8$ $(\mathrm{PE} / \mathrm{EtOAC}=4 / 1) .{ }^{1} \mathrm{H}$ NMR $\left(400 \mathrm{MHz}, \mathrm{CDCl}_{3}\right): \delta 4.82(\mathrm{~s}, 1 \mathrm{H}), 4.69(\mathrm{~s}, 1 \mathrm{H}), 3.69(\mathrm{~s}, 3 \mathrm{H}), 2.04-2.08(\mathrm{~m}$, 1H), 1.92-1.95 (m, 1H), $1.57(\mathrm{~s}, 3 \mathrm{H}), 1.30(\mathrm{~m}, 4 \mathrm{H}), 1.01(\mathrm{~s}, 3 \mathrm{H}), 0.89(\mathrm{~s}, 21 \mathrm{H}), 0.04(\mathrm{~s}, 6 \mathrm{H}),-0.06(\mathrm{~s}$, 6H). ${ }^{13} \mathrm{C}$ NMR $\left(100 \mathrm{MHz}, \mathrm{CDCl}_{3}\right): \delta 178.4,137.3,133.0,82.2,81.1,60.9,51.8,31.0,26.0(6 \mathrm{C}), 25.3$, 23.1, 18.3, 18.2, 14.2, 11.5, 11.4, -4.4, $-4.6,-4.7,-4.8 . \mathrm{HRMS}\left(\mathrm{ESI}^{+}\right) \mathrm{m} / \mathrm{z}:[\mathrm{M}+\mathrm{Na}]^{+}$calcd for $\mathrm{C}_{25} \mathrm{H}_{50} \mathrm{NaO}_{4} \mathrm{Si}_{2}$, 493.3140; found, 493.3120 .

$14 a$

yellow oil. $(144 \mathrm{mg}, 86 \%) R_{f}=0.6(\mathrm{PE} / \mathrm{EtOAC}=4 / 1) .{ }^{1} \mathrm{H} \mathrm{NMR}\left(400 \mathrm{MHz}, \mathrm{CDCl}_{3}\right): \delta 4.04-4.09(\mathrm{~m}, 2 \mathrm{H})$, 2.42-2.49 (m, 2H), $2.02(\mathrm{~s}, 3 \mathrm{H}), 1.43-1.47(\mathrm{~m}, 2 \mathrm{H}), 1.34(\mathrm{~s}, 3 \mathrm{H}), 1.28-1.31(\mathrm{~m}, 2 \mathrm{H}), 1.09(\mathrm{t}, J=8 \mathrm{~Hz}$, 3H), $0.86(t, J=8 \mathrm{~Hz}, 3 \mathrm{H}) .{ }^{13} \mathrm{C} \mathrm{NMR}\left(100 \mathrm{MHz}, \mathrm{CDCl}_{3}\right): \delta 199.8,199.5,166.6,159.9,155.4,62.2$, 57.5, 29.6, 24.0, 22.6, 15.0, 13.9, 13.8, 9.7. $\mathrm{HRMS}\left(\mathrm{ESI}^{+}\right) \mathrm{m} / \mathrm{z}:[\mathrm{M}+\mathrm{Na}]^{+}$calcd for $\mathrm{C}_{14} \mathrm{H}_{20} \mathrm{NaO}_{4}$, 275.1254; found, 275.1249 .

$14 \mathrm{c}$ 
yellow oil. $(282 \mathrm{mg}, 77 \%) R_{f}=0.6(\mathrm{PE} / \mathrm{EtOAC}=4 / 1) .{ }^{1} \mathrm{H} \mathrm{NMR}\left(400 \mathrm{MHz}, \mathrm{CDCl}_{3}\right): \delta 3.95-4.01(\mathrm{~m}, 2 \mathrm{H})$, 2.41-2.47 (m, 2H), $2.01(\mathrm{~s}, 3 \mathrm{H}), 1.41-1.44(\mathrm{~m}, 2 \mathrm{H}), 1.35(\mathrm{~s}, 3 \mathrm{H}), 1.26-1.31(\mathrm{~m}, 2 \mathrm{H}), 1.16-1.19(\mathrm{~m}$, $2 \mathrm{H}), 0.85(\mathrm{t}, J=8 \mathrm{~Hz}, 3 \mathrm{H}), 0.79(\mathrm{t}, J=8 \mathrm{~Hz}, 3 \mathrm{H}) .{ }^{13} \mathrm{CNMR}\left(100 \mathrm{MHz}, \mathrm{CDCl}_{3}\right): \delta 199.8,199.5,166.6$, $159.8,155.5,65.9,57.6,30.3,29.7,24.0,22.7,18.9,15.0,13.9,13.6,9.6 . \mathrm{HRMS}\left(\mathrm{ESI}^{+}\right) \mathrm{m} / \mathrm{z}:[\mathrm{M}+$ $\mathrm{Na}]^{+}$calcd for $\mathrm{C}_{16} \mathrm{H}_{24} \mathrm{NaO}_{4}, 303.1567$; found, 303.1563.

$14 d$

yellow oil. $(275 \mathrm{mg}, 83 \%) R_{f}=0.6(\mathrm{PE} / \mathrm{EtOAC}=4 / 1) .{ }^{1} \mathrm{H} \mathrm{NMR}\left(400 \mathrm{MHz}, \mathrm{CDCl}_{3}\right): \delta 3.58(\mathrm{~s}, 3 \mathrm{H}), 2.46$ $(\mathrm{t}, J=8 \mathrm{~Hz}, 2 \mathrm{H}), 2.40-2.10(\mathrm{~m}, 2 \mathrm{H}), 2.02(\mathrm{~s}, 3 \mathrm{H}), 1.42-1.47(\mathrm{~m}, 2 \mathrm{H}), 1.26-1.32(\mathrm{~m}, 2 \mathrm{H}), 0.86(\mathrm{t}, J=8$ $\mathrm{Hz}, 3 \mathrm{H}), 0.62(\mathrm{t}, \mathrm{J}=8 \mathrm{~Hz}, 3 \mathrm{H}) .{ }^{13} \mathrm{C} \mathrm{NMR}\left(100 \mathrm{MHz}, \mathrm{CDCl}_{3}\right): \delta 199.7,199.4,166.8,161.2,156.9,62.1$, 53.1, 29.8, 24.1, 23.5, 22.7, 13.9, 9.6, 8.5. HRMS (ESI $\left.{ }^{+}\right) \mathrm{m} / \mathrm{z}:[\mathrm{M}+\mathrm{Na}]^{+}$calcd for $\mathrm{C}_{14} \mathrm{H}_{20} \mathrm{NaO}_{4}$, 275.1254; found, 275.1258 .

$14 \mathrm{e}$

yellow oil. $(299 \mathrm{mg}, 81 \%) R_{f}=0.6(\mathrm{PE} / \mathrm{EtOAC}=4 / 1) .{ }^{1} \mathrm{H} \mathrm{NMR}\left(400 \mathrm{MHz}, \mathrm{CDCl}_{3}\right): \delta 4.01-4.05(\mathrm{~m}, 2 \mathrm{H})$, $2.46(\mathrm{t}, J=8 \mathrm{~Hz}, 2 \mathrm{H}), 2.01(\mathrm{~s}, 3 \mathrm{H}), 1.97-2.00(\mathrm{~m}, 2 \mathrm{H}), 1.42-1.46(\mathrm{~m}, 2 \mathrm{H}), 1.26-1.31(\mathrm{~m}, 2 \mathrm{H}), 1.09(\mathrm{t}$, $J=8 \mathrm{~Hz}, 3 \mathrm{H}), 0.88-0.93(\mathrm{~m}, 2 \mathrm{H}), 0.86(\mathrm{t}, J=8 \mathrm{~Hz}, 3 \mathrm{H}), 0.78(\mathrm{t}, J=8 \mathrm{~Hz}, 3 \mathrm{H}) .{ }^{13} \mathrm{C} \mathrm{NMR}(100 \mathrm{MHz}$, $\left.\mathrm{CDCl}_{3}\right): \delta 199.0,199.6,166.4,161.0,156.6,62.1,61.9,32.0,29.8,24.0,22.7,17.5,14.3,13.9$ 13.9, 9.6. HRMS $\left(E S I^{+}\right) \mathrm{m} / \mathrm{z}:[\mathrm{M}+\mathrm{Na}]^{+}$calcd for $\mathrm{C}_{16} \mathrm{H}_{24} \mathrm{NaO}_{4}, 303.1567$; found, 303.1560 .

$14 f$

yellow oil. $(286 \mathrm{mg}, 78 \%) R_{f}=0.6(\mathrm{PE} / \mathrm{EtOAC}=4 / 1) .{ }^{1} \mathrm{H} \mathrm{NMR}\left(400 \mathrm{MHz}, \mathrm{CDCl}_{3}\right): \delta 2.35-2.53(\mathrm{~m}, 2 \mathrm{H})$, $2.00(\mathrm{~s}, 3 \mathrm{H}), 1.41-1.47(\mathrm{~m}, 2 \mathrm{H}), 1.28(\mathrm{~s}, 14 \mathrm{H}), 0.86(\mathrm{t}, J=8 \mathrm{~Hz}, 3 \mathrm{H}) .{ }^{13} \mathrm{C} \mathrm{NMR}\left(100 \mathrm{MHz}, \mathrm{CDCl}_{3}\right): \delta$ 200.3, 200.0, 165.5, 159.6, 155.3, 83.0, 58.4, 29.8, 27.7, 24.0, 22.7, 14.7, 13.9, 9.6. HRMS (ESI ${ }^{+}$) $m / z:[\mathrm{M}+\mathrm{Na}]^{+}$calcd for $\mathrm{C}_{16} \mathrm{H}_{24} \mathrm{NaO}_{4}, 303.1567$; found, 303.1569 . 


\section{${ }^{1} \mathrm{H}$ and ${ }^{13} \mathrm{C}$ NMR Spectra of Compounds}

${ }^{1}$ H NMR Spectrum for compound $\mathbf{1 6}$

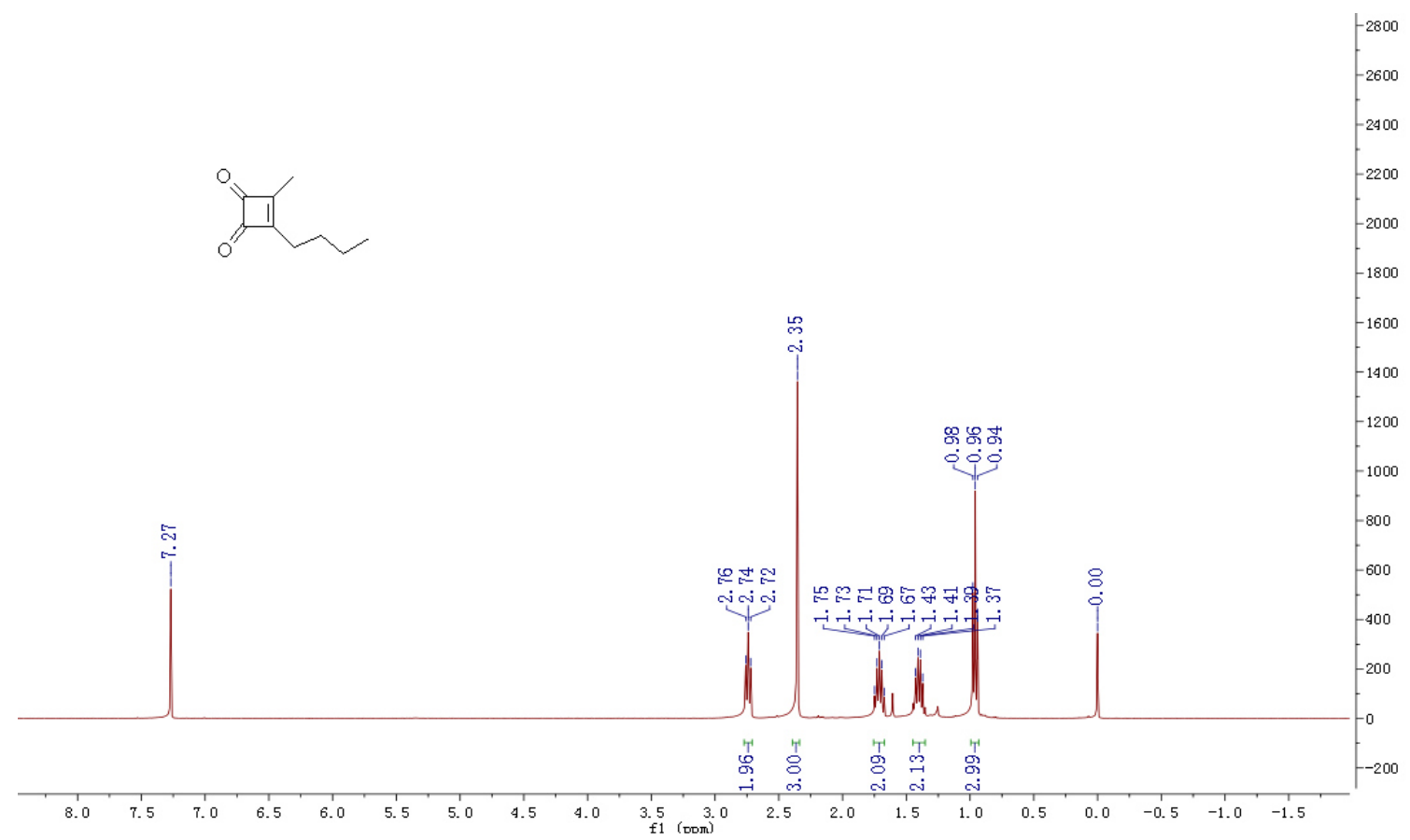

${ }^{13} \mathrm{C}$ NMR Spectrum for compound $\mathbf{1 6}$

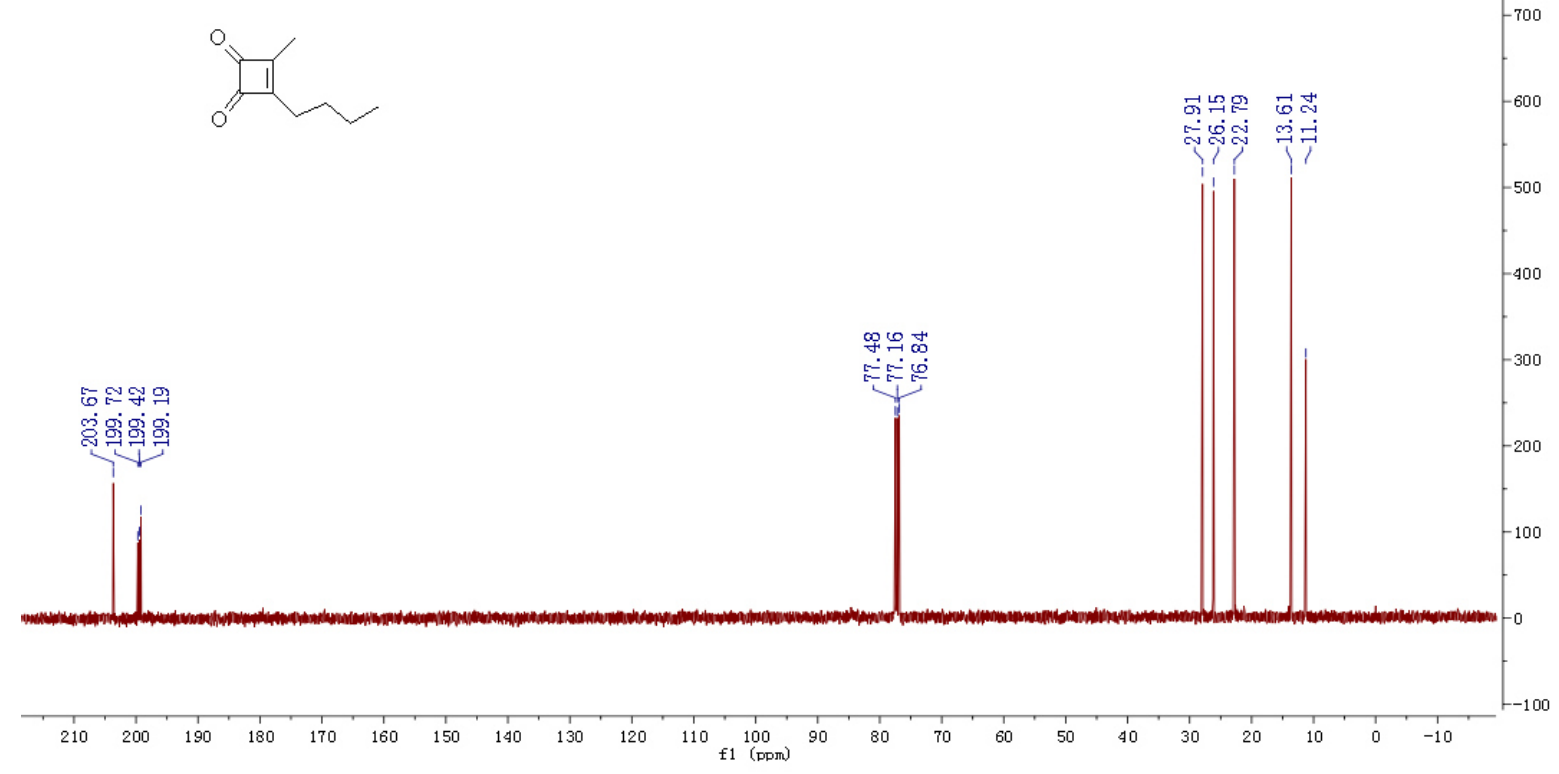


${ }^{1} \mathrm{H}$ NMR Spectrum for compound $\mathbf{1 4 b}$

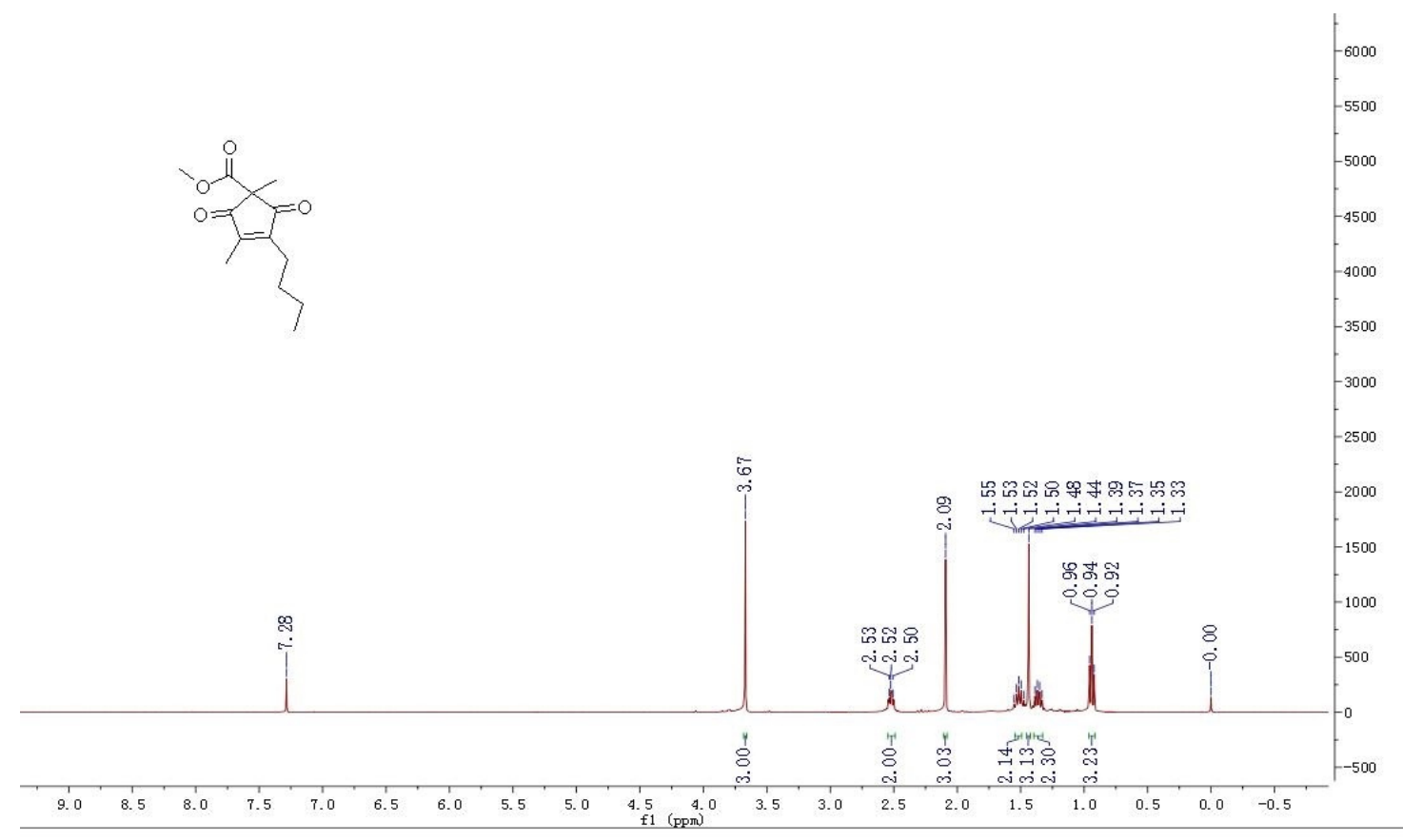

${ }^{13} \mathrm{C}$ NMR Spectrum for compound $\mathbf{1 4 b}$

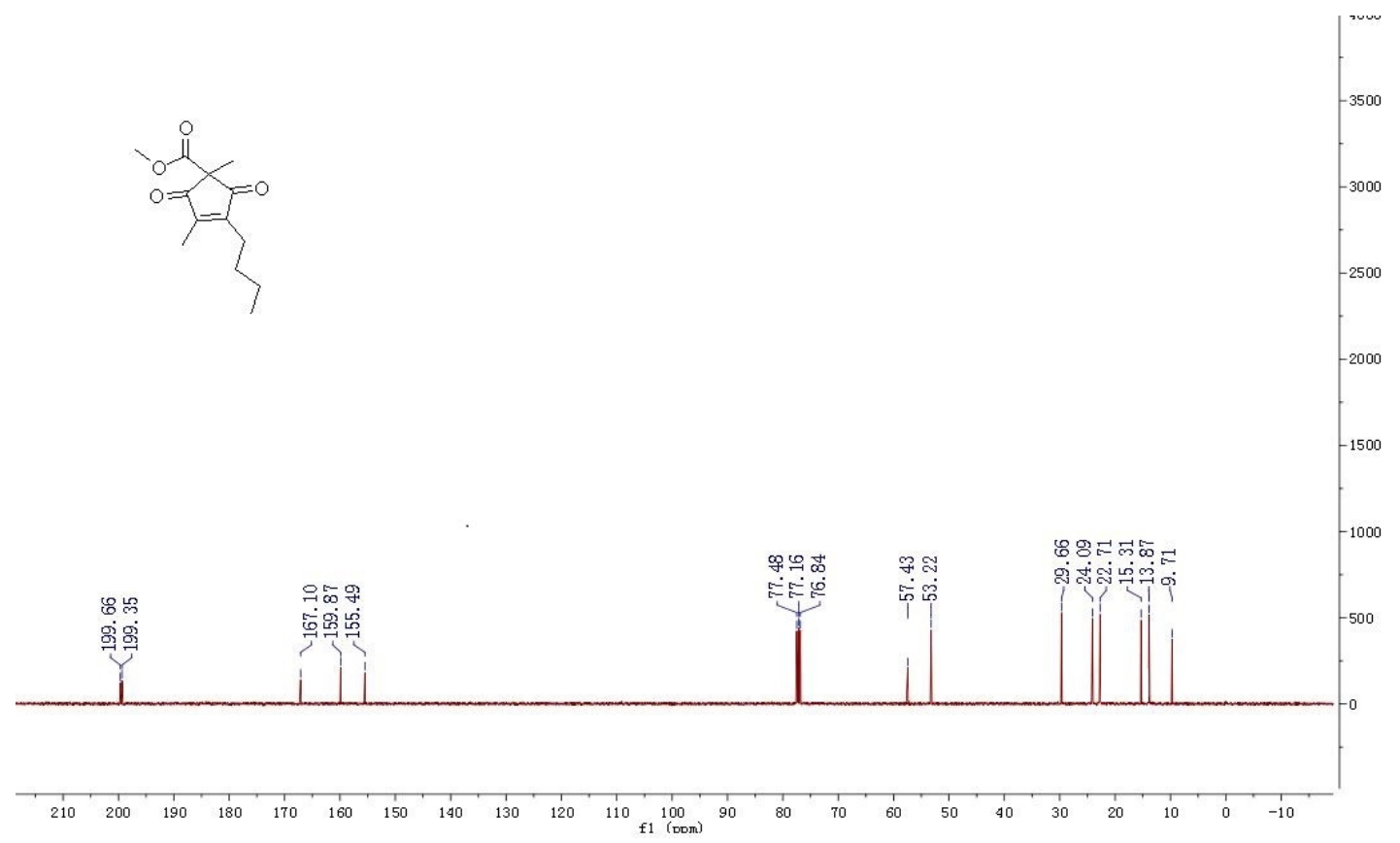


${ }^{1} \mathrm{H}$ NMR Spectrum for compound $\mathbf{1 3}$

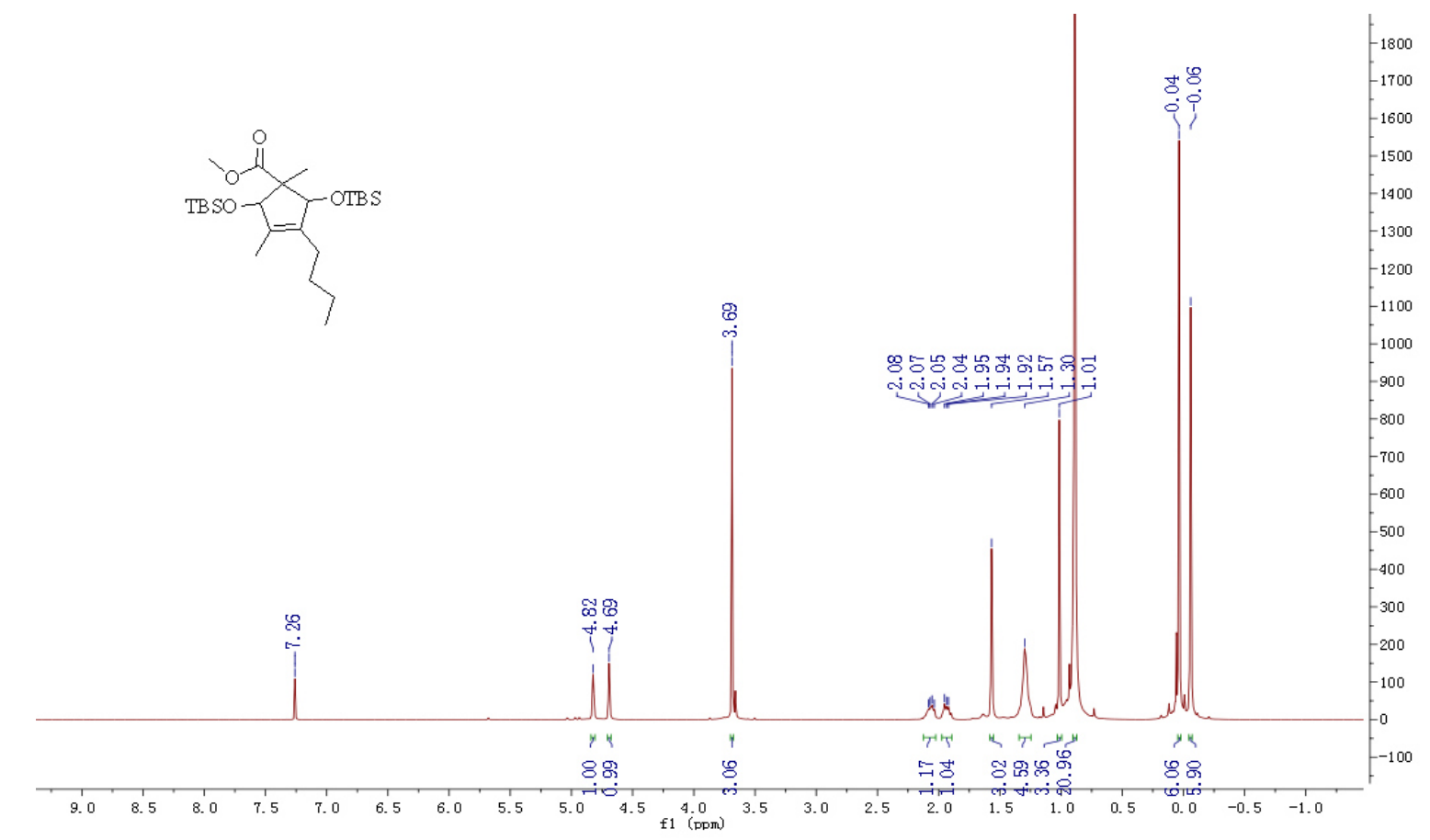

${ }^{13}$ C NMR Spectrum for compound 13

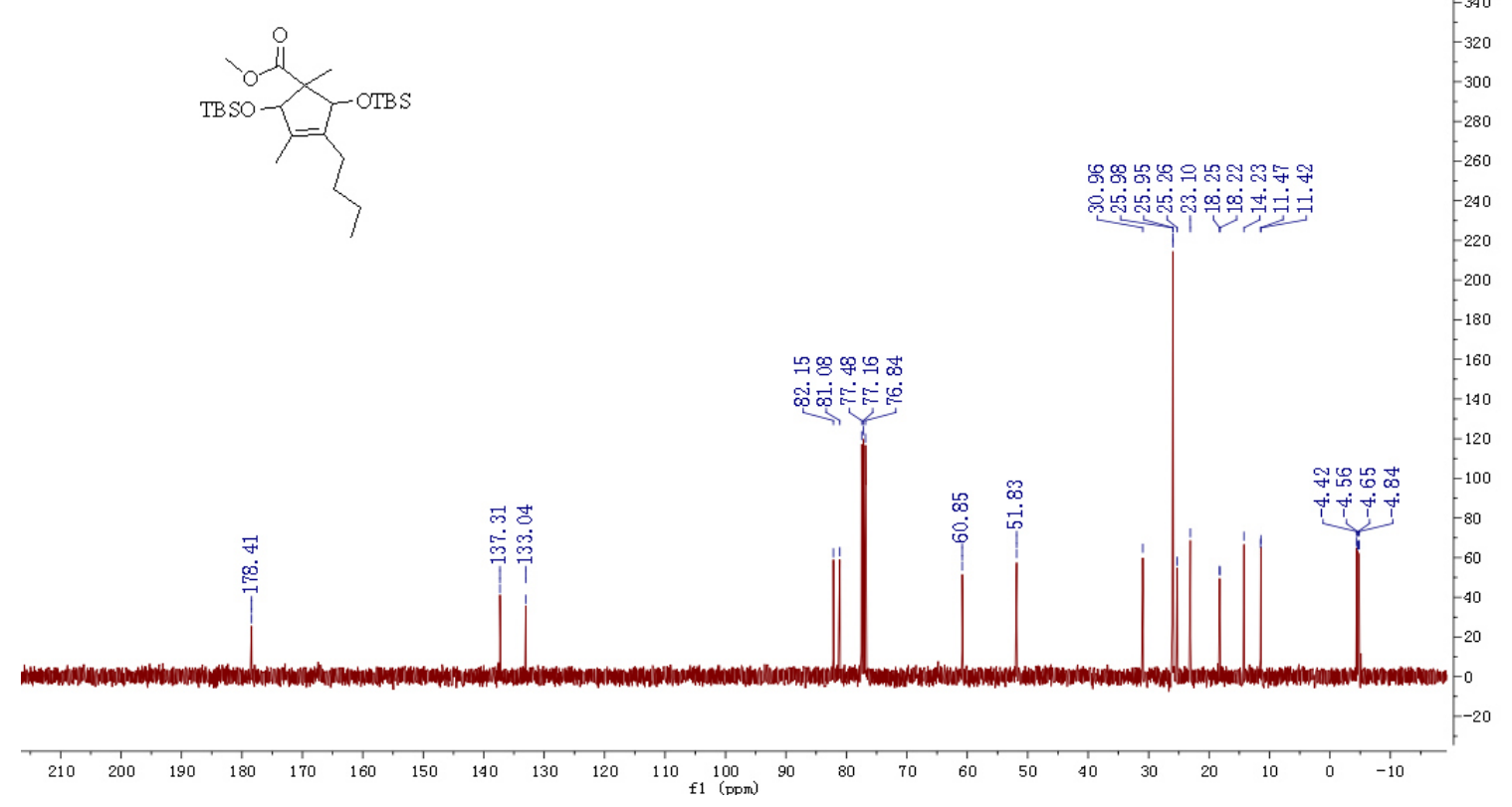


${ }^{1} \mathrm{H}$ NMR Spectrum for compound 14a

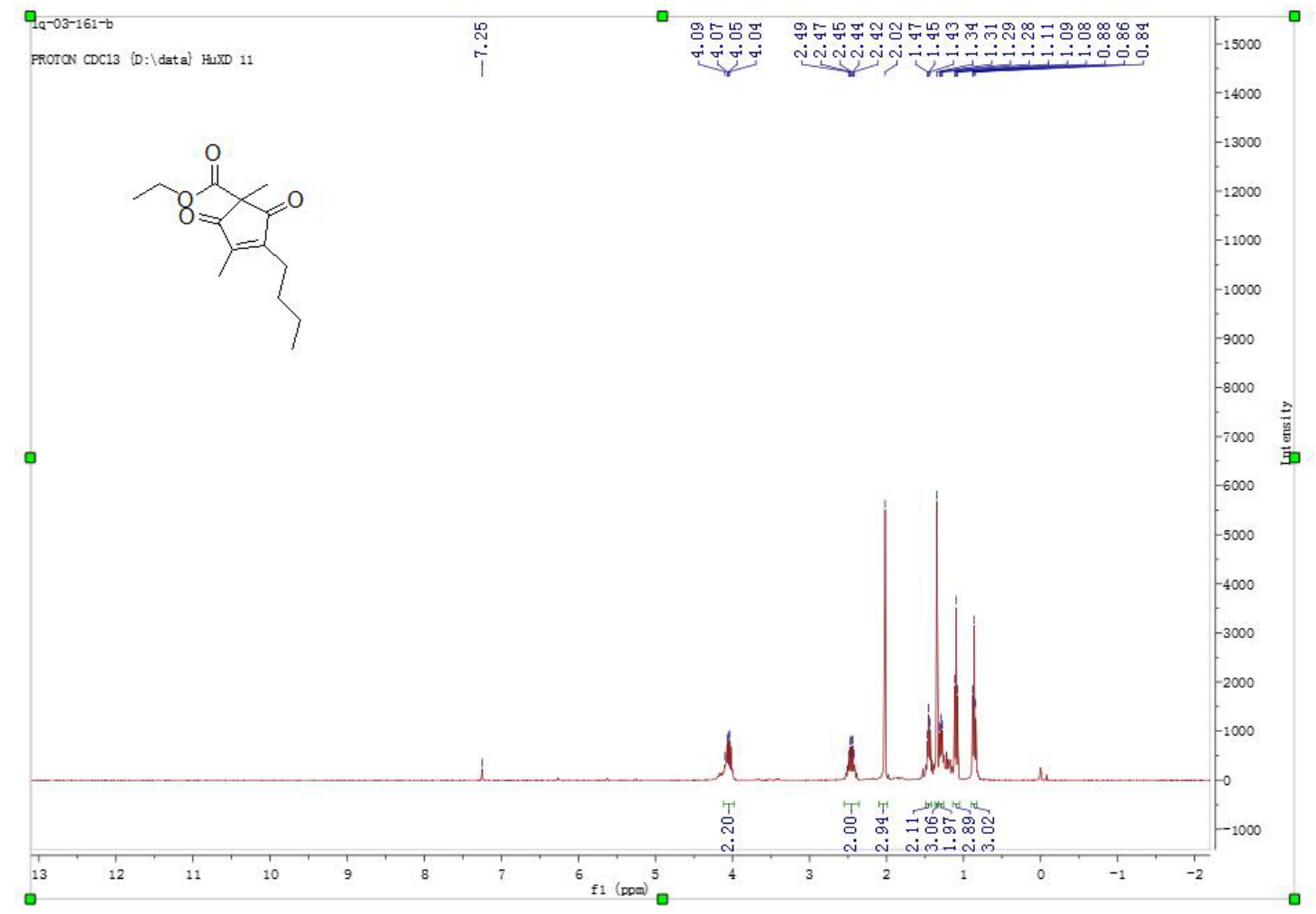

${ }^{13} \mathrm{C}$ NMR Spectrum for compound $\mathbf{1 4 a}$

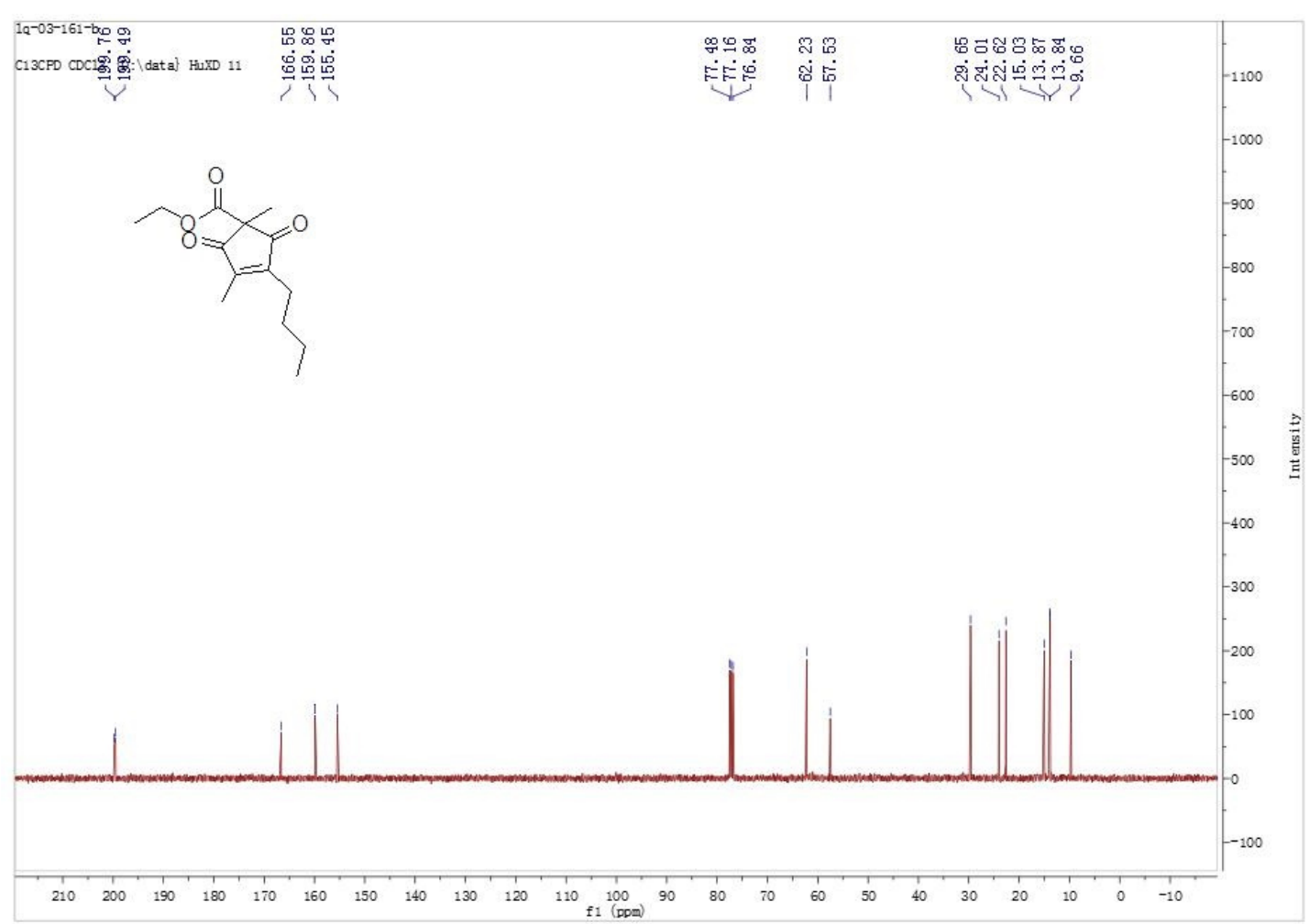


${ }^{1}$ H NMR Spectrum for compound $\mathbf{1 4 c}$

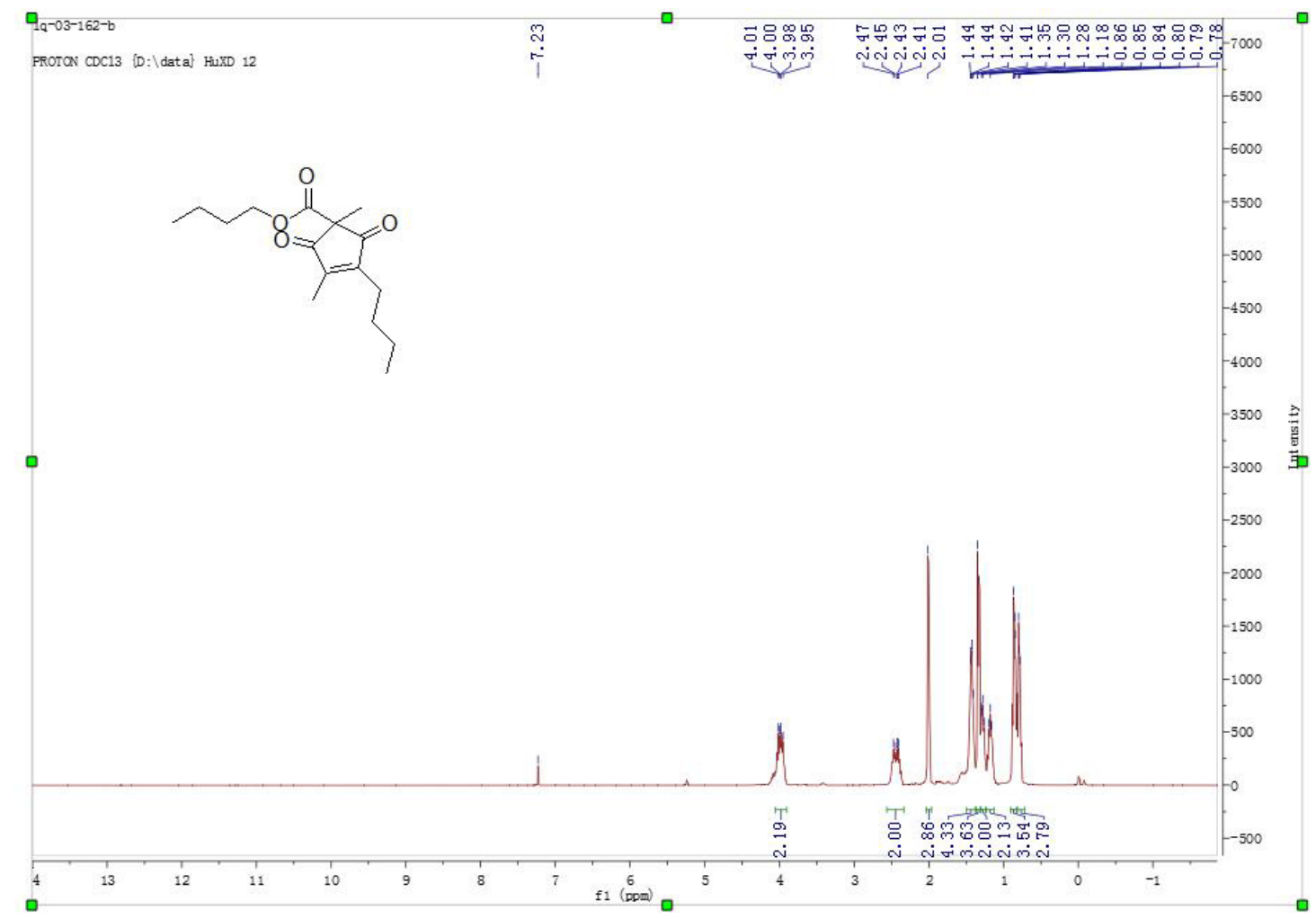

${ }^{13} \mathrm{C}$ NMR Spectrum for compound $\mathbf{1 4 c}$

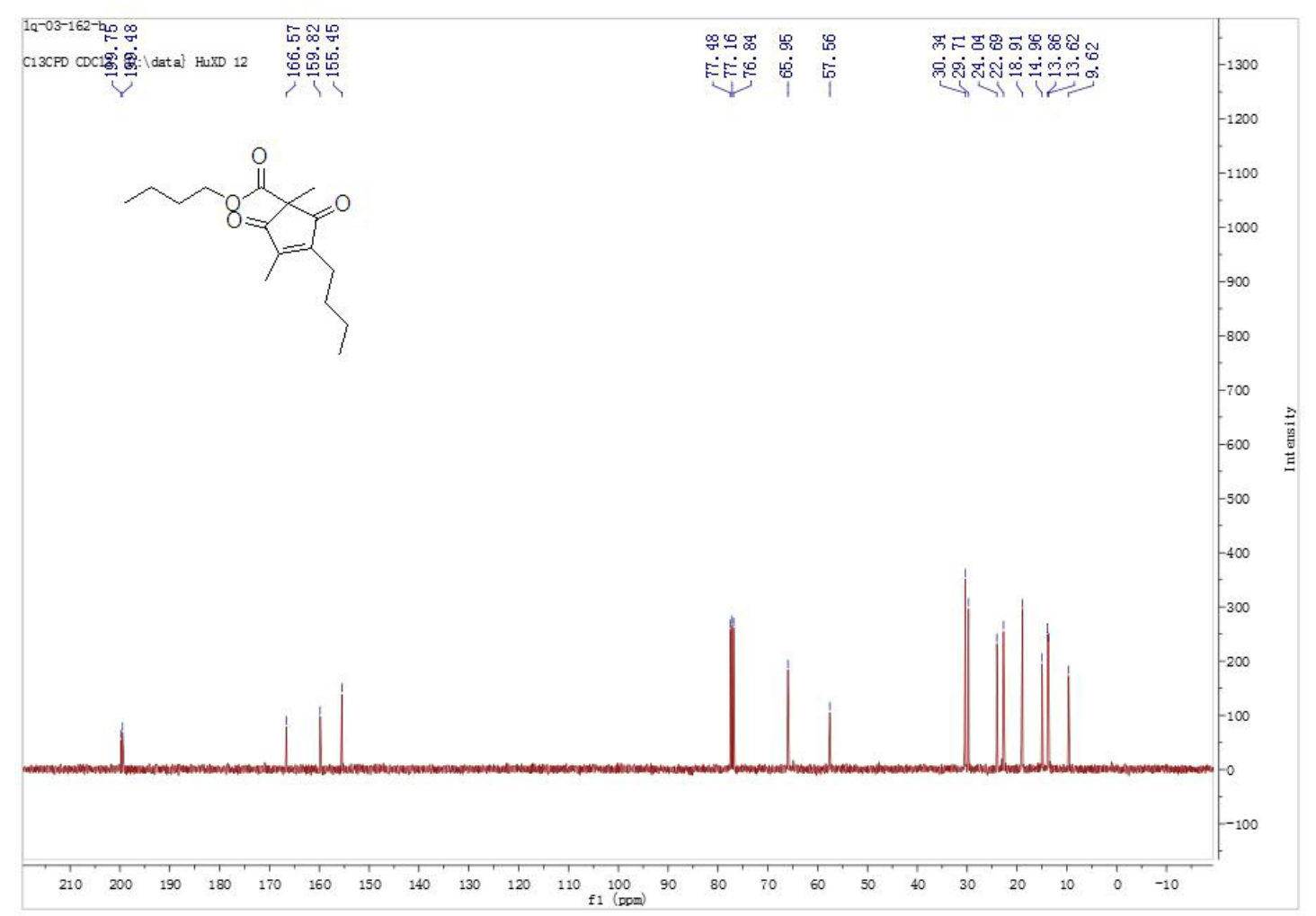


${ }^{1}$ H NMR Spectrum for compound 14d

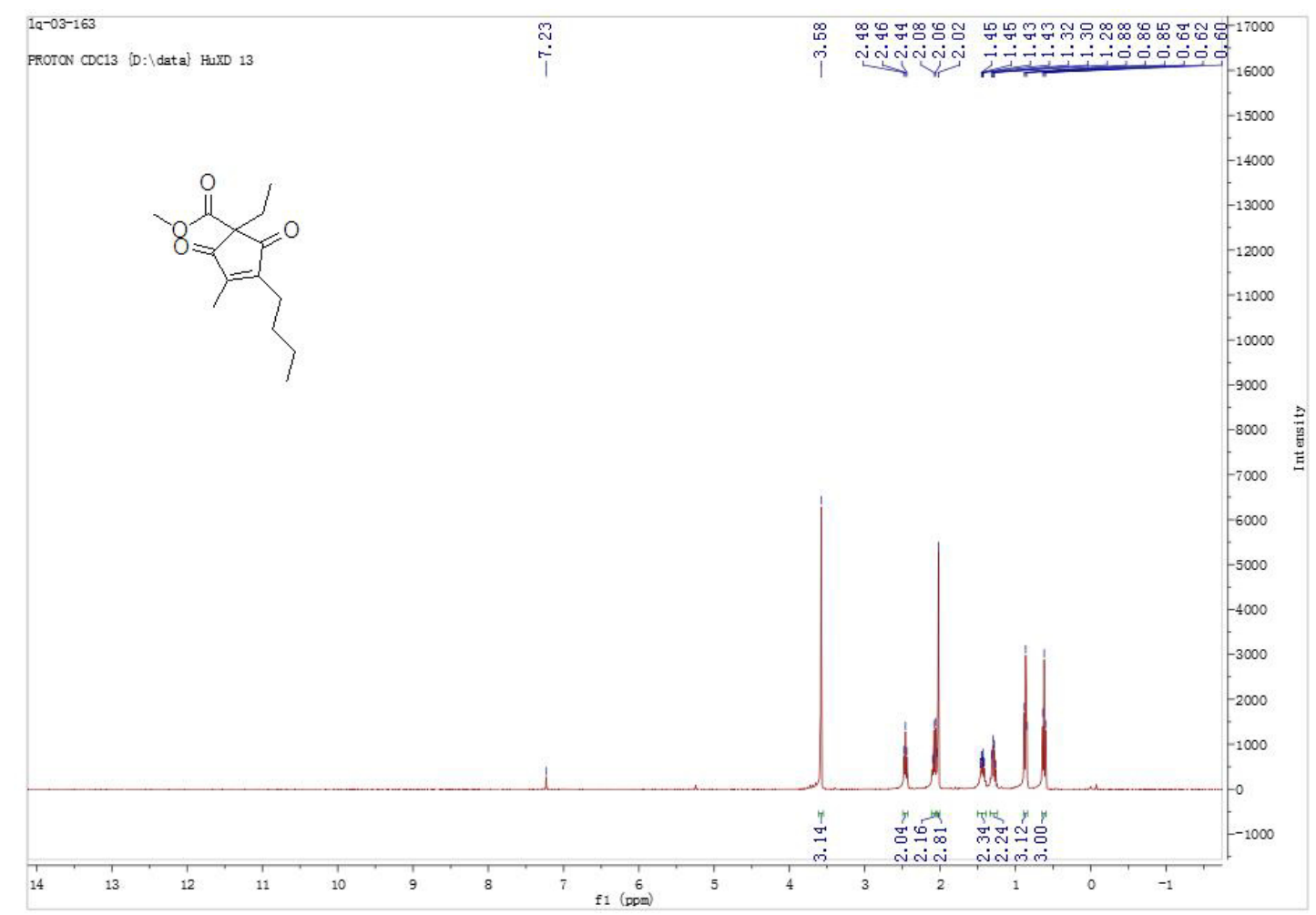

${ }^{13}$ C NMR Spectrum for compound $\mathbf{1 4 d}$

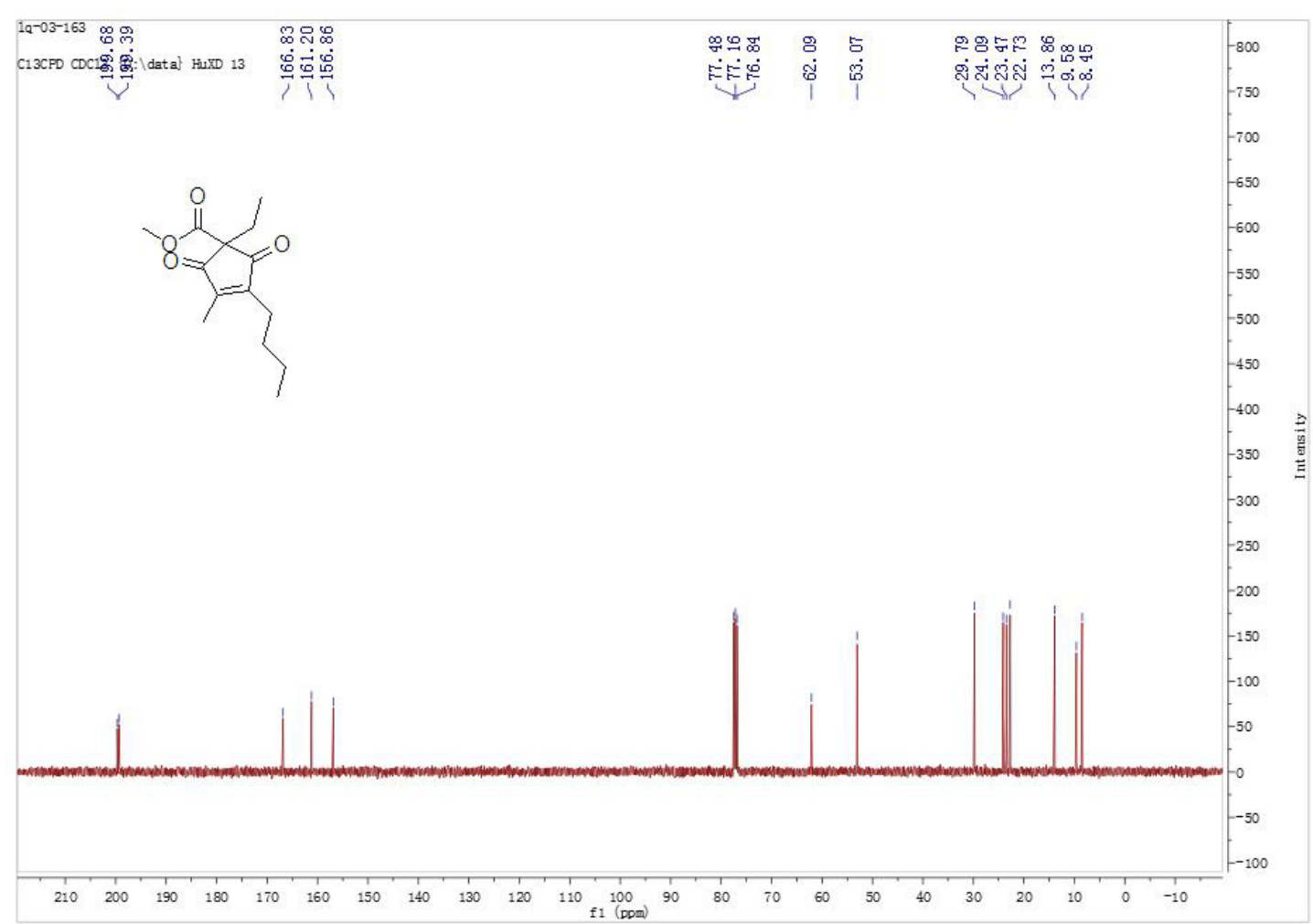


${ }^{1}$ H NMR Spectrum for compound $\mathbf{1 4 e}$

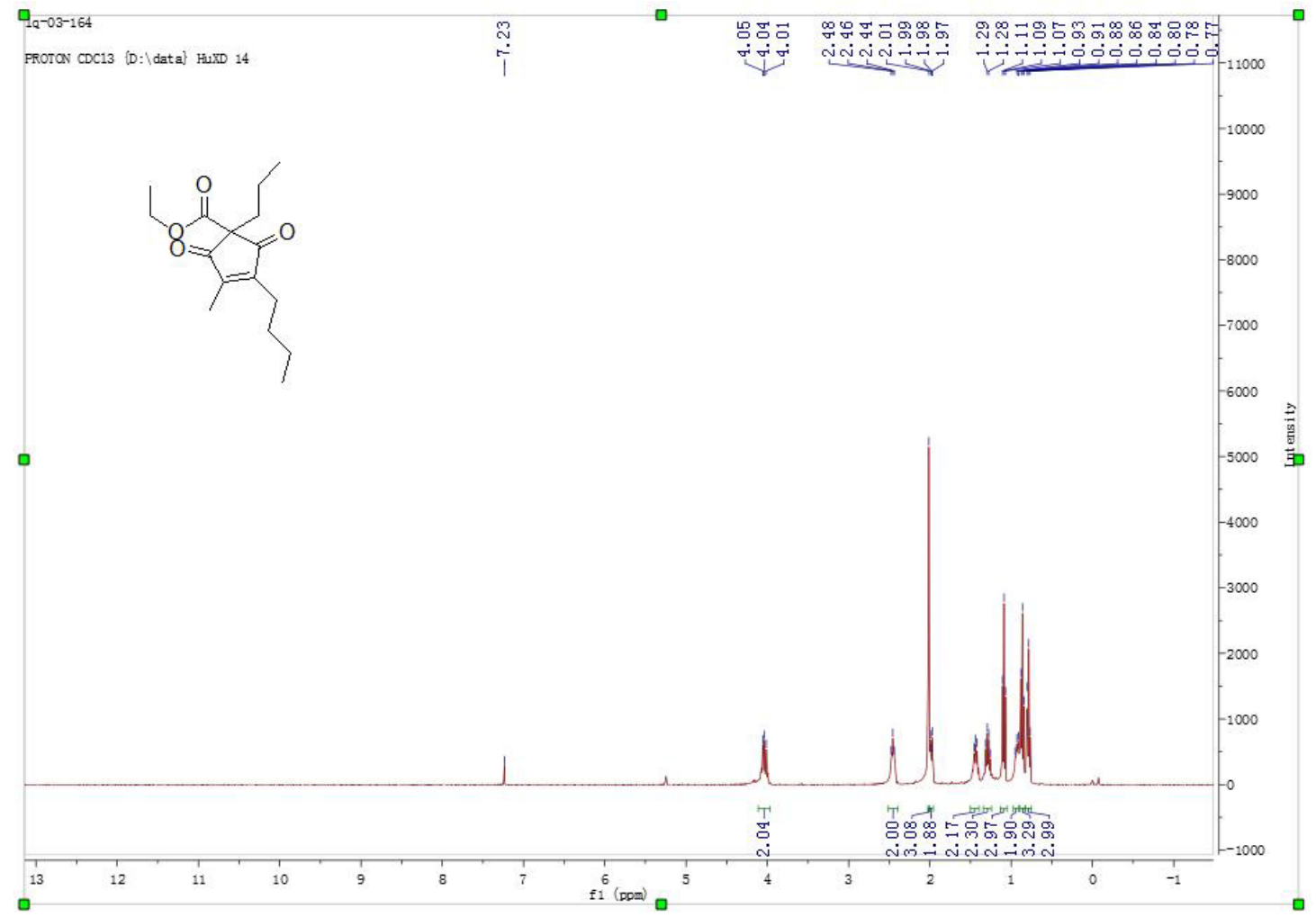

${ }^{13} \mathrm{C}$ NMR Spectrum for compound $\mathbf{1 4 e}$

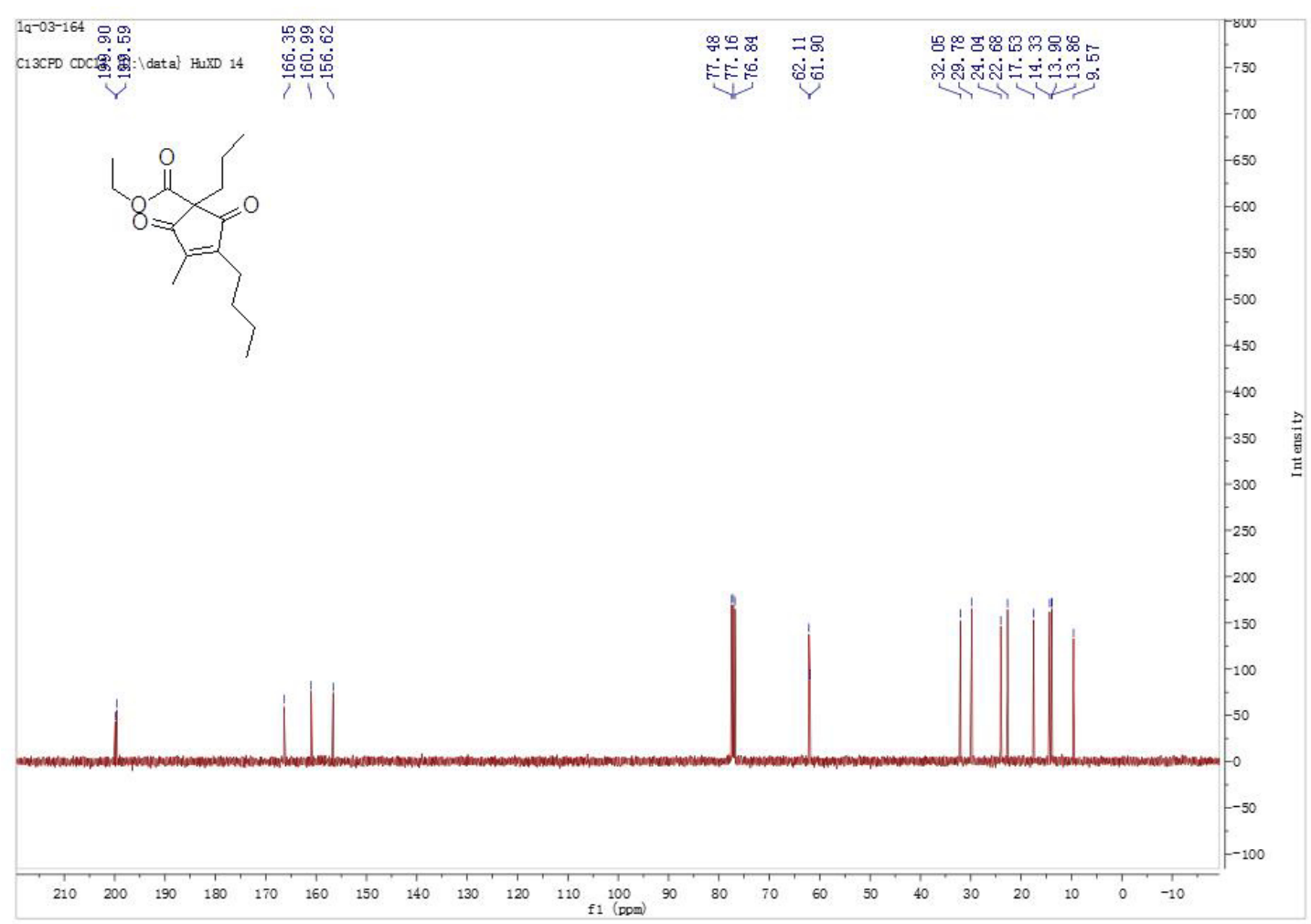


${ }^{1}$ H NMR Spectrum for compound $\mathbf{1 4 f}$

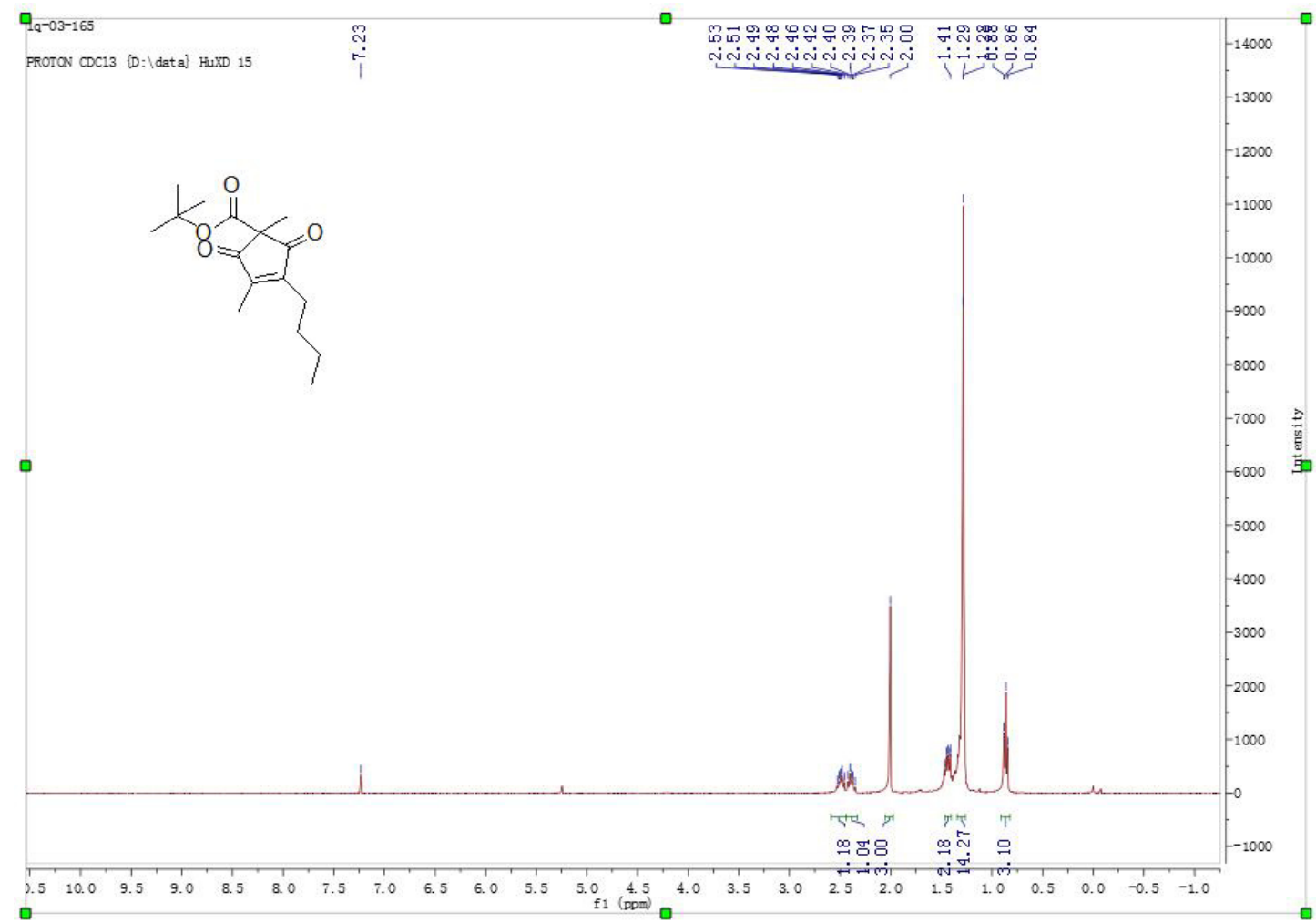

${ }^{13} \mathrm{C}$ NMR Spectrum for compound $\mathbf{1 4 f}$

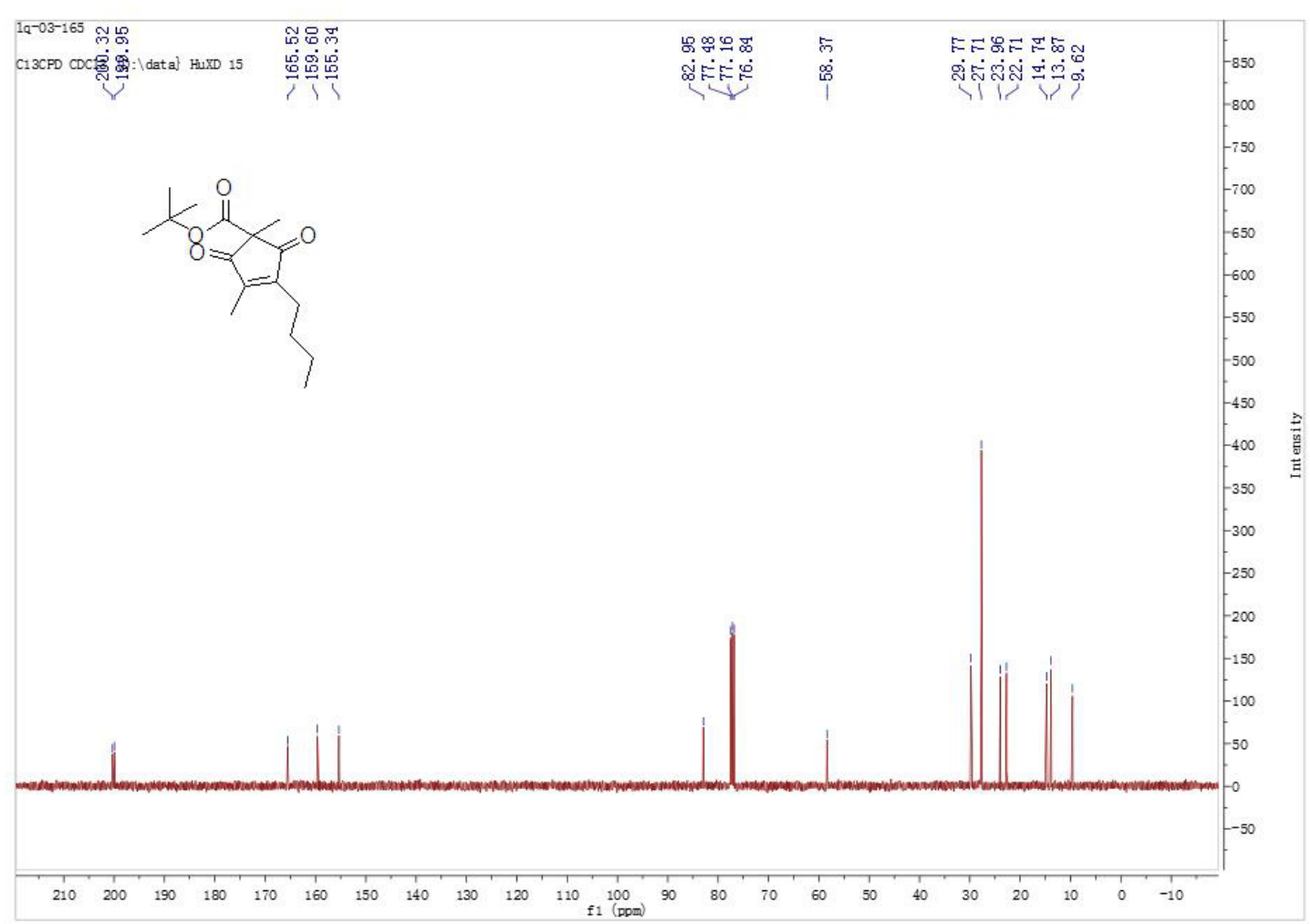

